\title{
Aplicación del análisis digital de imagen a los estudios de biodeterioro
}

\section{del Patrimonio Histórico}

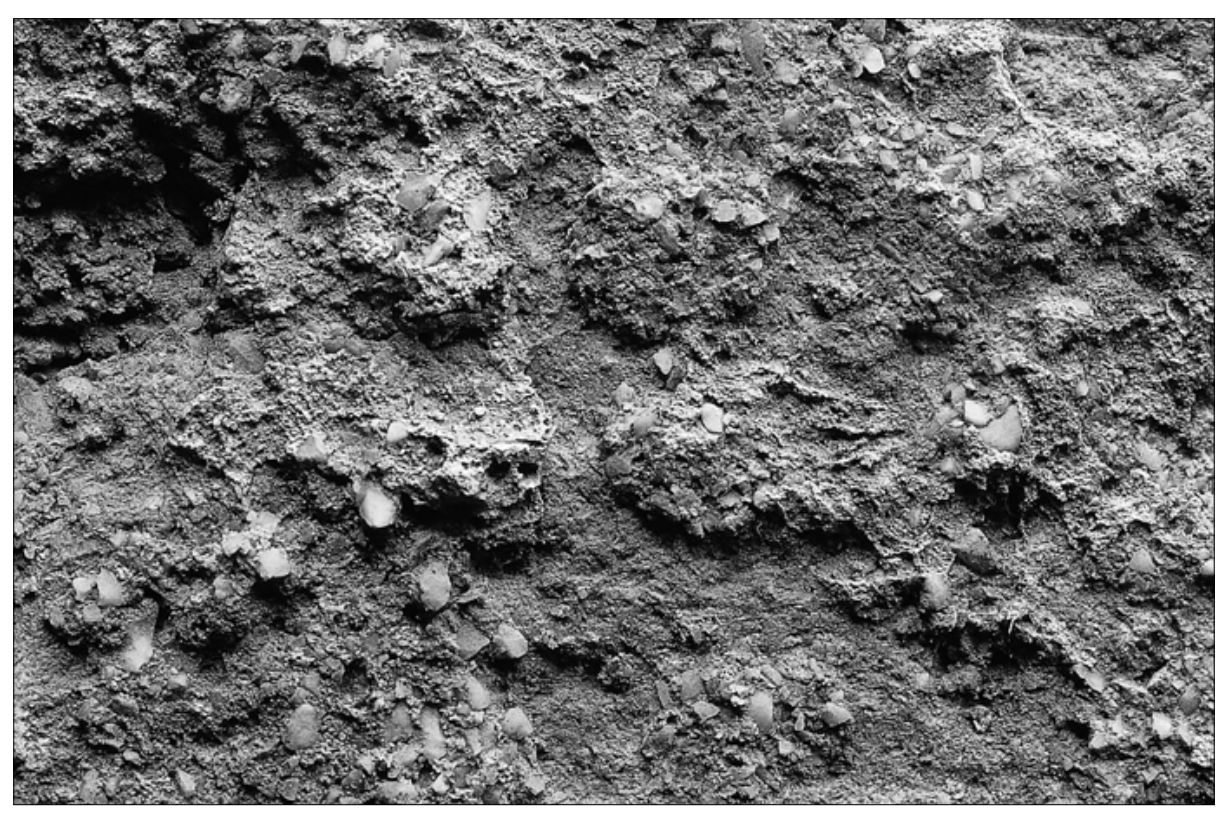

Antonio López Román Marta Sameño Puerto Centro de Intervención. Departamento de Análisis del IAPH.

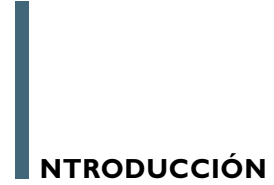

El empleo de las técnicas de análisis digital de imagen aplicadas dentro del ámbito del estudio del estado de los edificios históricos ha sido una herramienta interesante dentro de la metodología de análisis no destructivo. Hasta el momento su empleo se había centrado, fundamentalmente, en el estudio de características petrográficas macroscópicas o microscópicas, litotipos, porosidad, etc... de los materiales de construcción y cartografías de lesiones de edificios (Ángel, M.C. y cols., 1995).

Un aspecto interesante dentro del estudio de la posible degradación de los edificios históricos es el análisis de los factores relacionados con el biodeterioro. 
Se llama biodeterioro a las alteraciones que sufren los materiales orgánicos o inorgánicos por la influencia ya sea física o química de los organismos vivos que coexisten en el mismo ambiente.

Cualquier organismo puede afectar potencialmente una obra de arte, desde microorganismos como las bacterias hasta plantas superiores o vertebrados, pasando por hongos, líquenes, insectos, etc... El deterioro de los materiales pétreos de los edificios, yacimientos arqueológicos y esculturas aumenta progresivamente con el paso del tiempo (Allsop, D. Y K.J. Seal, 1986). Una de las mayores causas de este proceso es la acción de los vegetales tanto vasculares como no vasculares ( G. Caneva y cols, 1993).

Las piedras con las que se construyen los edificios históricos constituyen un buen sustrato de crecimiento para el desarrollo de algas, líquenes, musgos, y finalmente plantas superiores en un gradiente temporal que sigue el orden que hemos mencionado anteriormente. Cuando la humedad del sustrato es permanente se desarrollan predominantemente las cianobacterias, algas y briofitos (G. Caneva y cols, 1994), sin embargo si la humedad no es permanente se desarrollan fundamentalmente los líquenes de biotipo crustáceo con una influencia clara en la alteración de dicho sustrato.

Los estudios sobre líquenes y musgos en el patrimonio histórico son muy amplios. Son organismos de crecimiento lento, necesitan pocos requerimientos ecológicos y presentan diferentes formas y colores (ICR-CNR, 1985) y son capaces de colonizar prácticamente cualquier ambiente por hostil que sea para el resto de las formas de vida (M.R.D. Seaward y C. Giacobini, 1988). Son los primeros colonizadores biológicos de las piedras de los monumentos.

Los estudios sobre líquenes en el patrimonio histórico se extienden tanto en lo referente a su ecología y sociabilidad como en lo que respecta a los procedimientos de eliminación más seguros, sin afectar al sustrato sobre el que se desarrollan, para evitar alteraciones debidas al uso de los productos más que a la presencia de los organismos.

Estos estudios se basan fundamentalmente en una caracterización de la flora presente en la zona de estudio (número y denominación de las especies presentes, índice de abundancia) en relación con otros parámetros ambientales (grado de humedad, tipo de sustrato, presencia de otros organismos, etc). El análisis de los datos revela información valiosa sobre el comportamiento de estos organismos frente a los materiales con los que está construida la obra, justificando de una manera global su 'bioalteración', en el caso de que exista, o su 'bioprotección' frente a los agentes fisico-químicos atmosféricos agresivos para la misma.

Muchos de los estudios que se realizan tienen como fin una eliminación selectiva de este material biológico utilizando biocidas más o menos específicos. La utilización de biocidas se suele llevar a cabo realizando pruebas es- paciales localizadas y analizando la eficacia de los mismos frente al organismo que se quiere eliminar utilizando como parámetro el grado de desaparición del organismo.

Aunque los análisis que se han descrito anteriormente son clásicos, el abordaje de los mismos problemas, mediante la utilización de técnicas nuevas, puede revelar datos interesantes sobre aspectos desconocidos hasta el momento en los estudios del comportamiento de los organismos vivos y su influencia sobre el deterioro de los materiales con los que se construyen los monumentos.

En este trabajo se pretenden presentar dos aplicaciones del análisis digital de imagen a los casos que relacionan los organismos vivos con los procesos de alteración de los monumentos, dentro del estudio global que se está llevando a cabo en el Castillo de Mairena del Alcor. Por un lado, un procedimiento sencillo de cuantificación y construcción de un mapa de distribución y abundancia de diferentes especies de líquenes presentes en algunas muestras de las murallas del castillo, útil para los estudios de ecología y sociabilidad de estas criptógamas, y por otro la propuesta de un sistema de seguimiento temporal y análisis objetivo del efecto de varias sustancias biocidas sobre una superficie afectada por el crecimiento de algas.

\section{MATERIAL Y MÉTODO}

\section{Presencia de líquenes}

El procedimiento que se siguió para el estudio de la presencia de los líquenes fue el que a continuación se expone. Se eligieron diferentes zonas donde se realizó un inventario de los líquenes presentes. Se definieron las características del área, tipo de sustrato, orientación, inclinación, procurando que las zonas de inventario elegidas abarcasen todas las posibles variabilidades en cada uno de estos parámetros.

Las zonas en las que se realizó el muestreo se fotografían y se determinan las especies de líquenes presentes en cada una de ellas mediante observación 'in situ' y mediante análisis en el laboratorio utilizando diferentes técnicas (lupa binocular, microscopio óptico, uso de reactivos, etc.) y bibliografía especializada (Ozenda, P. y G. Clauzade, 1970).

Los líquenes presentan formas y colores característicos. Una vez que se han identificado y determinado todas las especies presentes en la zona de estudio hay que proceder a aislarlas unas de otras informáticamente.

Sobre la imagen digitalizada optimizada se determina la gama de colores que se corresponde con cada una de las especies.

Esa imagen se binariza mediante un proceso de segmentación y se extrae la información correspondiente a cada una de las áreas ocupadas por las especies pre- 
sentes. Como la imagen se encuentra referenciada espacialmente es muy fácil obtener cual es el porcentaje de la superficie segmentada dentro del total de la imagen. De este modo y teniendo en cuenta que las zonas donde se realizan los inventarios no siempre tienen el mismo tamaño, el resultado final nunca, en ninguno de los ensayos, se deforma ya que los datos se representan en forma de porcentaje.

De igual forma, en el caso de que se requiera conocer exactamente el área que ocupa cada uno de los líquenes también se puede representar con unidades eliminando de la representación porcentual.

\section{Determinación de la efectividad de biocidas}

Los ensayos con biocidas se llevaron a cabo siguiendo el procedimiento que, a continuación, se detalla.

En primer lugar se define cual es la zona sobre la que se va a realizar el ensayo. La zona en cuestión ha de presentar una cubierta de organismos lo más homogénea posible, con objeto de minimizar las posibles desviaciones de las muestras que se han de analizar.

La zona donde se realicen las pruebas ha de ser pequeña para evitar variaciones bruscas de alguno de los parámetros ambientales, de modo que el crecimiento de los organismos no se vea afectado, salvo por la acción de la sustancia biocida.

Dentro de la zona de análisis se han de marcar áreas más pequeñas y de similar tamaño donde se van a aplicar los biocidas. En la figura 2, A se muestra la zona de estudio antes de la aplicación de los biocidas con las áreas donde se ensaya cada una de las sustancias.

Una vez aplicado cada biocida en su zona correspondiente se deja transcurrir el tiempo para comprobar la eficacia del tratamiento.

Antes de la aplicación de las sustancias biocidas se ha de fotografiar la zona. En los intervalos de tiempo en los que se decida que se va a comprobar el efecto del tratamiento se ha de volver a fotografiar la zona desde el mismo punto, con objeto de minimizar los errores debidos a un cambio de perspectiva. De igual forma es necesario que dentro de la imagen aparezca una escala que nos proporcione una dimensión exacta de la superficie real de la zona analizada.

Partimos de la base que sólo las algas, que intentamos identificar y que presentan un color verde, se encuentran en un estado vegetativo saludable.

Las imágenes tomadas en intervalos de tiempo diferentes y digitalizadas se llevan a una escala similar con objeto de que la comparación de las superficies ocupadas por las plantas antes y después del tratamiento sea lo más objetiva posible.

Una vez optimizada la imagen procede a extraer la información correspondiente a la gama de los verdes que se corresponde a la clorofila de los vegetales presentes en el terreno mediante un procedimiento llamado segmentación de la imagen. La imagen así obtenida es una imagen binaria sobre la que se puede calcular que porcentaje de la misma ha sufrido el proceso de selección mediante el color.

De igual forma se procede a realizar el proceso de segmentación en la zona equivalente de la foto correspondiente a las zonas tratadas. De igual manera obtendremos un área seleccionada por el color verde que se corresponde con un porcentaje determinado frente al área total analizada.

En este caso los datos obtenidos también se muestran porcentualmente, por la inmediatez que se obtiene en la evaluación de los resultados finales.

\section{Tratamiento de la imágenes}

Las imágenes obtenidas a través del registro fotográfico se digitalizan utilizando un escaner. En este momento las imágenes analógicas, en soporte fotográfico, se transforman en imágenes digitales. Las imágenes digitales se han de tratar mediante diferentes técnicas de procesado digital para optimizarlas en función de los parámetros que necesitamos analizar y de los datos que queremos extraer.

\section{RESULTADOS Y DISCUSIÓN}

\section{Inventario de líquenes}

A partir de la imagen original digitalizada (fig. I, A), se aplicó la técnica del 'falso color' (fig. I, B) para contrastar la imagen que se iba analizar. El resultado del proceso originó una imagen irreal de la zona en la que se realizó el inventario de las especies presentes. El hecho de que la imagen nos parezca irreal es irrelevante a la hora de realizar el análisis digital de la imagen, ya que la información contenida en el fichero correspondiente es de interés para extraer datos de la misma.

Nosotros presentamos un ejemplo del trabajo realizado, sin embargo no todas las imágenes requieren el mismo tratamiento. Sobre cada imagen hay que realizar el procedimiento de optimización de la información digital que sea más adecuado para extraer los datos que interesan.

Una vez que la imagen ha sido optimizada se extraen los datos correspondientes a cada una de las especies presentes.

En el estudio 'in situ' y de laboratorio de la zona que presentamos en este trabajo se han detectado cuatro especies diferentes de líquenes de biotipo crustáceo: Verrucaria tabacina, Aspicilia coronata, Lecanora dispersa y Caloplaca variabilis y uno foliáceo Xantoria aureola. 
En la figura I se presentan los resultados del análisis de la zona donde se realizó el inventario que se presenta.

En la figura I, A se muestra en color negro a imagen original de la zona de estudio. La zona debe estar referenciada espacialmente como ya hemos comentado con objeto de conocer cual es la escala sobre la que evaluar los datos. La figura I, B muestra la imagen digital optimizada con falsos colores.

En la figura I C-G se muestra en color negro las zonas ocupadas por cada una de las especies.

En la figura I, H se muestra en color negro el mapa de las zonas no colonizadas por líquenes, es decir, zonas donde se manifiesta el sustrato o zonas donde no se ha identificado correctamente ninguna de las especies que se han identificado.

En la tabla I se muestra el porcentaje del área ocupada por cada una de las especies con respecto a la zona de estudio total.

Tabla I. Especies de líquenes presentes en la zona y superficie porcentual ocupada por cada una de ellas después de su análisis.

\begin{tabular}{lc}
\hline \multicolumn{1}{c}{ ESPECIE } & $\%$ DEL ÁREA OCUPADA \\
\hline Xantoria aureola (Arch.) Erichs. & 34.43 \\
\hline Aspicilia coronata (Massal.) B. De Lesd. & 0.473 \\
\hline Caloplaca variabilis (Pers.) Müll. Arg & 2.025 \\
\hline Lecanora dispersa (Pers.) Röhl & 7.133 \\
\hline Verrucaria tabacina (Massal.) Trev. & 11.728 \\
\hline Sustrato no colonizado & 44.205 \\
\hline TOTAL & 100 \\
\hline
\end{tabular}

La zona colonizada por todas las especies de líquenes identificadas ocupa un área del $55.795 \%$ de la zona de estudio, es decir, más de la mitad estaba ocupada por organismos vivos.

En la figura I, C se muestra el área de distribución de Xantoria aureola . La distribución de esta especie ocupa un gran área en la zona de estudio, el $34.43 \%$. Los talos se encuentran distribuidos en una gran masa central con crecimientos periféricos, pero prácticamente en contacto todos los talos.

En I, D se muestra el área de distribución de la especie Aspicilia coronata. Como se puede apreciar la superficie relativa ocupada por esta especie es mucho menor que la colonizada por Xantoria aureola, en concreto esta especie ocupa el $0.473 \%$ de la zona de estudio . Los talos no están en contacto entre sí, encontrandose de un modo disperso por toda la zona donde se realiza el inventario. De igual forma el creci-
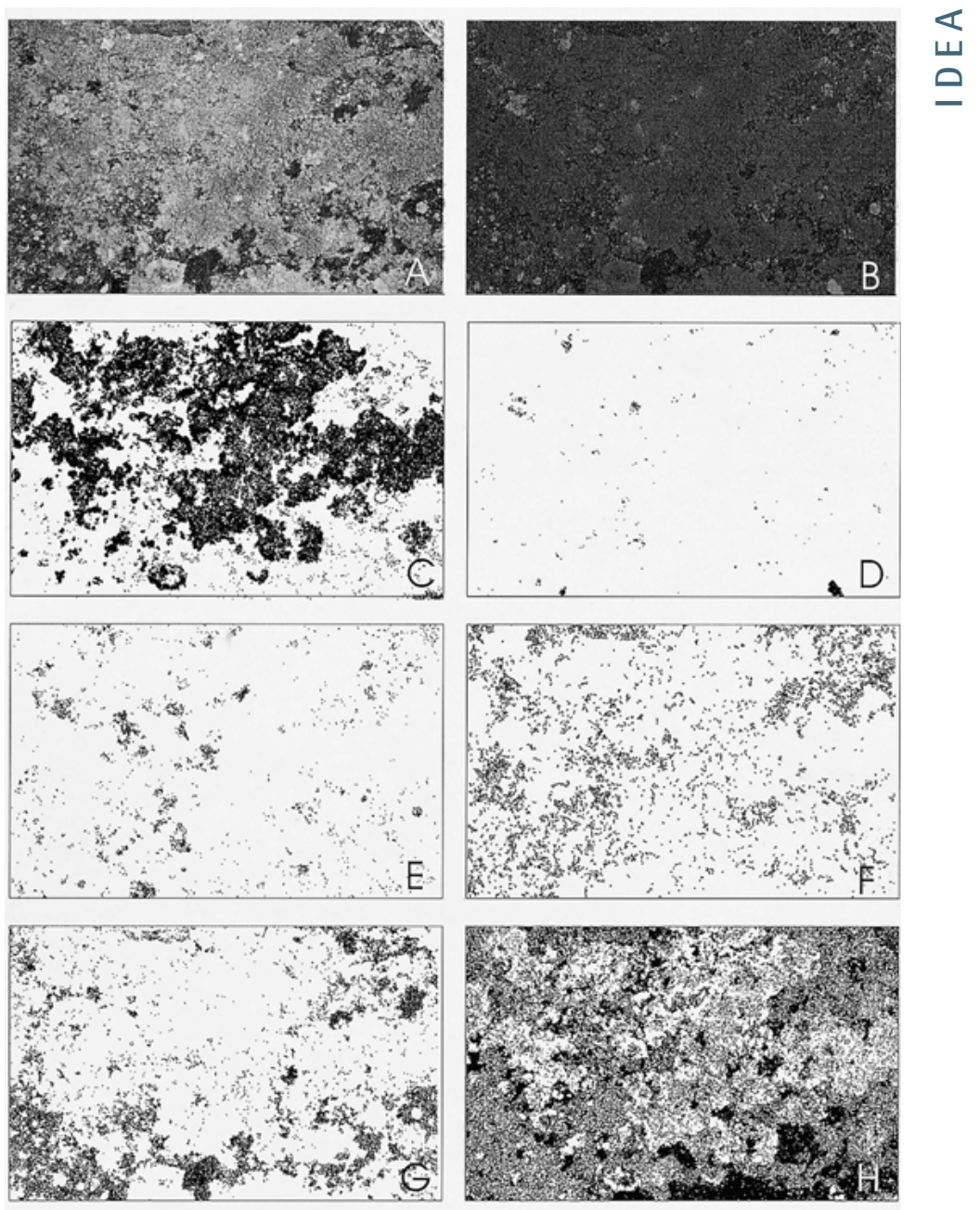

Figura I
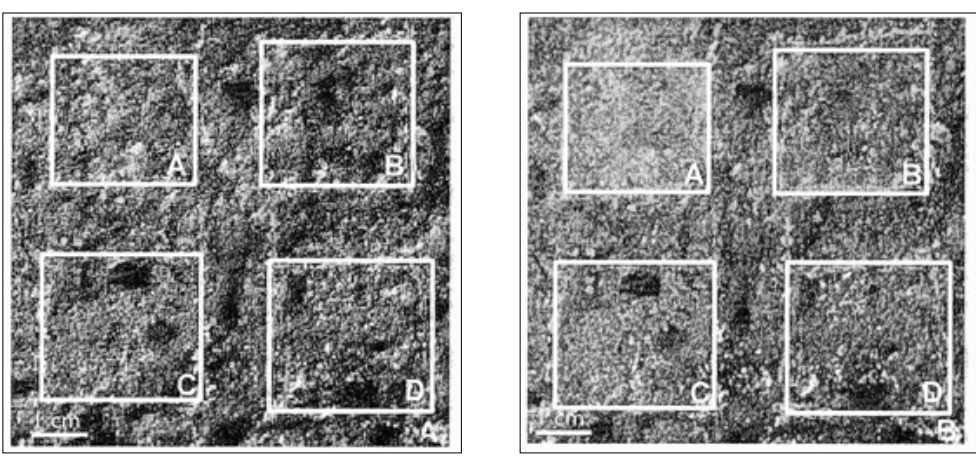

Figura 2

miento no se realiza colonizando grandes áreas, sino que los crecimientos son muy localizados y ocupando pequeñas superficies.

En la figura I, E se presenta el área de desarrollo de Caloplaca variabilis. La superficie relativa ocupada es pequeña (2.025 \%). La distribución de la especie es bastante homogénea, aunque su crecimiento se restringe a zonas pequeñas, la expansión de sus talos es limitada.

En la figura I, F se muestra la zona colonizada por Lecanora dispersa. Su distribución ocupa prácticamente
Figura I. Presencia de líquenes en las murallas del castiIlo. A. Fotografía original. B. Imagen digital optimizada.

Mapas de distribución. C. Xantoria aureola. D. Aspicilia coronata E. Caloplaca variabilis. F. Lecanora dispersa. G.Verrucaria tabacina. $\mathrm{H}$. Zonas sin presencia liquénica.

Figura 2. Supeficie de la piedra tratada con sustancias biocidas. A. Antes del tratamiento. B. Una semana después del tratamiento. 
toda la zona de estudio, pero no presenta grandes talos que invadan todo el sustrato. Como se aprecia en la figura su dispersión es difusa, ocupando una superficie del $7.133 \%$.

En la figura I, G se presenta el área de distribución de Verrucaria tabacina. La presencia de este líquen es considerable dentro del cómputo global de cada una de las especies, ocupando una superficie del II.728\% del total. Las masas de crecimiento de esta especie se encuentran en relación estrecha, es decir, no crecen de un modo aislado.

En estos resultados se aprecia como la distribución de las especies se encuentra perfectamente definida, aislada una de otra, cómo se demuestra en la figura I. Con la información que se puede extraer del análisis de estos datos se puede estudiar la forma de crecimiento de las especies de líquenes presentes en el sustrato si se realizan fotografías periódicamente de la zona, la sociabilidad de los mismos en sus relaciones vitales, la supervivencia de algunas especies frente a otras en el mismo terreno, la tasa de crecimiento a lo largo del tiempo como índice objetivo de capacidad de colonización de estos organismos en los edificios históricos, la capacidad de resistencia frente a agentes liquenicidas y otra serie de procesos relacionados con las dinámicas relaciones que se establecen entre este tipo de organismos y los sustratos que colonizan.

\section{Pruebas con biocidas}

Otro de los ensayos que hemos realizado es la del análisis de la efectividad de diferentes sustancias biocidas sobre un sustrato colonizado por algas.

Los ensayos con biocidas son una de las pruebas clásicas que se realizan para evaluar la capacidad de resistencia de algunos organismos a su eliminación mediante la utilización de algunas sustancias que presentan alta toxicidad para los organismos que se quieren eliminar y, normalmente, también para el hombre. Existe gran cantidad de sustancias con capacidad para erradicar los organismos vivos que se desarrollan en las obras de arte.

El ánimo de este estudio no es evaluar rigurosamente la naturaleza del biocida, de modo que las sustancias biocidas las vamos a denominar A, B, C y $D$. Cada una de estas sustancias se emplea en el área $A, B, C$ y $D$ respectivamente, con objeto de simplificar al máximo el planteamiento del problema y mostrar de una manera clara los resultados obtenidos mediante el uso de la metodología que se presenta.

En la figura 2 se muestran dos fotografías de la superficie de piedra expuesta al tratamiento con las cuatro sustancias biocidas antes citadas. La figura 2,A muestra la zona antes el tratamiento, mientras que la zona 2, B muestra la zona una semana después de que el biocida haya actuado.
El área de ensayo se encuentra delimitada para cada una de las sustancias empleadas por un marco de color blanco con su letra correspondiente para su mejor identificación.

Como se puede apreciar en la figura 2 las áreas de ensayo de los biocidas no son exactamente iguales en tamaño, debido a que en los trabajos de campo la precisión en la delimitación de las zonas es difícil. En concreto estos ensayos se marcaron sobre el terreno directamente mediante punción y en estas condiciones es difícil que todas las zonas marcadas sean idénticas. Además es irrelevante en este caso que las áreas de ensayo sean iguales ya que con una aproximación del tamaño de las mismas los efectos de los biocidas se aprecian perfectamente.

Con objeto de poder comparar las alteraciones en cuanto a la supervivencia de los organismos frente a los biocidas, cada una de las fotos, que se realizaron con un lapso de tiempo apreciable, de una semana, desde la misma posición.

Con este sencillo procedimiento se pueden comparar qué superficie de algas en estado vegetativo se encontraban presentes en el terreno antes y después el tratamiento. En función de los valores obtenidos conoceremos en cuanto ha disminuido el porcentaje de desarrollo de los organismos y por lo tanto, como veremos más adelante, el grado de eficacia del biocida.

Como todas las zonas de ensayo no son del mismo tamaño nos vemos obligados a mostrar los datos obtenidos en porcentaje. Realmente esta medida es más intuitiva a la hora de realizar las comparaciones y además elimina el problema que se podría plantear por la diferencia los tamaños de las áreas marcadas para el ensayo. Los resultados obtenidos se muestran en la tabla II.

En cada una de las cuatro zonas de ensayo (A, B, C, D) la superficie ocupada por los organismos presentes oscila entre el 75 y el $89 \%$, la zona se encuentra colonizada fundamentalmente por algas.

En la zona tratada con el biocida A (figura 2,A,A y $2, B, A$ ) se aprecia a simple vista como existe una disminución apreciable de la cantidad de organismos biológicos presentes. De hecho una vez realizado el análisis digital podemos asegurar que existe una disminución del $27.87 \%$ en la superficie ocupada por las plantas. En la zona tratada con el biocida B, se muestra una disminución del $27.89 \%$ en la superficie que los organismos vivos colonizan. En la zona tratada con el biocida $C$ se aprecia una disminución del $22.6 \%$ en la superficie colonizada por las algas, mientras que en la zona tratada con el biocida $D$ la disminución de la población de algas es insignificante.

De este modo podemos concluir de una manera objetiva que tanto el biocida A como el B son los más potentes en este sustrato y para eliminar estos organismos, mientras que el efecto del biocida $D$ es inapreciable, luego su uso es el menos indicado. 
Tabla II. Porcentaje de la superfice de microorganismos afectada por el tratamiento con biocidas.

\begin{tabular}{lrrrc}
\hline & Superficie A & Superficie B & Superficie C & Superficie D \\
\hline Superficie Control (Fig. ,A) & $78.208 \%$ & $75.81 \%$ & $89.764 \%$ & $83.54 \%$ \\
\hline Superficie Tratada (Fig.2,B) & $56.407 \%$ & $54.66 \%$ & $69.477 \%$ & $83.2 \%$ \\
\hline Porcentaje de eliminación de organismos & $27.87 \%$ & $27.89 \%$ & $22.600 \%$ & $0.26 \%$ \\
\hline
\end{tabular}

En el procedimiento que se ha mostrado solamente se evalúa la superficie de sustrato, en este caso perteneciente a las murallas del Castillo de Mairena del Alcor, colonizada por organismos vivos, desde un enfoque relacionado con la posible alteración que pueden sufrir los monumentos. En ningún caso se puede aportar ningún dato sobre la desaparición de biomasa del mismo sustrato.

Como se ha podido demostrar el método que aquí se presenta es fácil y rápido de realizar, proporciona resultados objetivos y además se puede hacer un seguimiento temporal del grado de efectividad del biocida, no solo obteniendo datos de la eficacia de los biocidas a largo plazo, sino que se pueden analizar otros parámetros como son la rapidez de actuación de estas sustancias, o la estabilización de las poblaciones de organismos.

\section{CONCLUSIONES}

En este trabajo se presenta de una manera breve una metodología de análisis no destructivo para realizar estudios de biodeterioro del patrimonio histórico en dos supuestos prácticos diferentes.

Una de las ventajas principales de este método es que el análisis es muy rápido y los resultados son muy precisos.
Los datos que habitualmente son cualitativos, según la subjetividad de observador, se pueden parametrizar y por lo tanto hacerlos objetivos, con las interesantes implicaciones cientíícas que conlleva esta afirmación.

El análisis digital de imagen permite realizar una evaluación temporal continua de la zona de estudio sin afectar en absoluto la evolución de los procesos que ahí se desarrollen ya que el único requerimiento que se necesita es un aporte de información gráfica del área de estudio bien en formato analógico (fotografía, diapositivas, etc) como digital (registro en video, fotografía digital, etc).

Por los resultados expuestos anteriormente las técnicas de análisis digital de imagen aplicada a los estudios de biodeterioro pueden tener tanta relevancia en los procedimientos de análisis no destructivo como en este área del patrimonio como la ha tenido en el estudio petrográfico.

En estos dos ejemplos se presenta sólo una aproximación inicial a la gran cantidad de ensayos que se pueden realizar sobre poblaciones de organismos vivos que permanentemente se encuentran estableciendo relaciones dinámicas entre ellas y con las estructuras sobre las que se desarrollan, pudiendo ser reflejadas de una manera objetiva y por lo tanto obtenerse resultados analíticos con una base científica más sólida.

Bibliografía

ALLSOPP, D. Y K. J. SEAL ( 1986). Introduction to biodeterioration. Edward Arnold, London.

ÁNGEL, M.C. Y COLS. (1995). "Evaluación por tratamiento digital de imágenes del deterioro de monumentos. Metodología". Materiales de construcción, Vol. 45, n² 240, pag. 37- 46.

CANEVA, G., G. DE MARCO Y M.A. PONTRANDOLFI (1993). "Plant communities on the walls of Venosa castle (Basilicata, Italy) as biodeteriogens and bioindicators". Conservation of stone and other materials: Vol. I, 263-270. (M.J.Thiel). Rilem, London.
ICR-CNR (1985). Microflora autotrofa ed eterotrofa: tecniche di indagine visiva. Normal 19/85.

OZENDA, P. Y G. CLAUZADE (1970). Les lichens. Étude biologique et flore illustrée. Masson et Cie, París.

SEAWARD M. D. Y C. GIACOBINI (1988). Lichen-induced biodeterioration of italian monuments, frescoes and other archaeological materials. Lichens and monuments. C.N.R. Centro di studio. "Cause di deperimento e metodi di conservazione delle opere d'arte".- Roma. P. L. Nimis \& M. Monte, Trieste.

CANEVA, G., M. P. NUGARI Y O. SALVADORI (1994). La biologia nel Restauro. Nardini, Firenze. 